\title{
Article \\ Preoperative Predictors of Adverse Clinical Outcome in Emergent Repair of Acute Type A Aortic Dissection in 15 Year Follow Up
}

\author{
Miriam Freundt ${ }^{1,2, *, \dagger}$, Philipp Kolat ${ }^{1, \dagger}$, Christine Friedrich ${ }^{1}$, Mohamed Salem ${ }^{1}\left(\mathbb{D}\right.$, Matthias Gruenewald ${ }^{3}$, \\ Gunnar Elke ${ }^{3}{ }^{\circledR}$, Thomas Pühler ${ }^{1}{ }^{\circledR}$, Jochen Cremer $^{1}$ and Assad Haneya ${ }^{1}$ \\ 1 Department of Cardiovascular Surgery, University Medical Center Schleswig-Holstein, Campus Kiel, \\ Arnold-Heller-Straße 3, 24105 Kiel, Germany; Philipp.Kolat@uksh.de (P.K.); \\ Christine.Friedrich@uksh.de (C.F.); Mohamed.Salem@uksh.de (M.S.); Thomas.Puehler@uksh.de (T.P.); \\ Jochen.Cremer@uksh.de (J.C.); assad.haneya@uksh.de (A.H.) \\ 2 Heart and Vascular Institute, Intensive Care Unit, Penn State Health Milton S. Hershey Medical Center, \\ Hershey, PA 23538, USA \\ 3 Department of Anaesthesiology and Intensive Care Medicine, University Medical Center Schleswig-Holstein, \\ Campus Kiel, Arnold-Heller-Str. 3 Haus R3, 24105 Kiel, Germany; Matthias.Gruenewald@uksh.de (M.G.); \\ Gunnar.Elke@uksh.de (G.E.) \\ * Correspondence: mfreundt@psu.edu \\ + These authors contributed equally.
}

check for updates

Citation: Freundt, M.; Kolat, P.; Friedrich, C.; Salem, M.; Gruenewald, M.; Elke, G.; Pühler, T.; Cremer, J.; Haneya, A. Preoperative Predictors of Adverse Clinical Outcome in Emergent Repair of Acute Type A Aortic Dissection in 15 Year Follow Up. J. Clin. Med. 2021, 10, 5370. https://doi.org/10.3390/jcm10225370

Academic Editor: Michał Ciurzyński

Received: 1 October 2021

Accepted: 16 November 2021

Published: 18 November 2021

Publisher's Note: MDPI stays neutral with regard to jurisdictional claims in published maps and institutional affiliations.

Copyright: (c) 2021 by the authors. Licensee MDPI, Basel, Switzerland. This article is an open access article distributed under the terms and conditions of the Creative Commons Attribution (CC BY) license (https:// creativecommons.org/licenses/by/ $4.0 /)$.
Abstract: Background: Acute type A aortic dissection (AAAD) has high mortality. Improvements in surgical technique have lowered mortality but postoperative functional status and decreased quality of life due to debilitating deficits remain of concern. Our study aims to identify preoperative conditions predictive of undesirable outcome to help guide perioperative management. Methods: We performed retrospective analysis of 394 cases of AAAD who underwent repair in our institution between 2001 and 2018. A combined endpoint of parameters was defined as (1) 30-day versus hospital mortality, (2) new neurological deficit, (3) new acute renal insufficiency requiring postoperative renal replacement, and (4) prolonged mechanical ventilation with need for tracheostomy. Results: Total survival/ follow-up time averaged 3.2 years with follow-up completeness of $94 \%$. Endpoint was reached by $52.8 \%$. Those had higher EuroSCORE II (7.5 versus 5.5), higher incidence of coronary artery disease (CAD) (9.2\% versus 3.2\%), neurological deficit (ND) upon presentation ( $26.4 \%$ versus $11.8 \%)$, cardiopulmonary resuscitation (CPR) $(14.4 \%$ versus $1.6 \%)$ and intubation (RF) before surgery ( $16.9 \%$ versus $4.8 \%$ ). 7 -day mortality was $21.6 \%$ versus $0 \%$. Hospital mortality $30.8 \%$ versus $0 \%$. Conclusions: This 15-year follow up shows, that unfavorable postoperative clinical outcome is related to ND, CAD, CPR and RF on arrival.

Keywords: predictor; adverse outcome; emergent surgical repair; acute type A dissection

\section{Introduction}

Acute type A aortic dissection (AAAD) is a catastrophic event in which the inner layer of the ascending aorta tears and separates from the middle layer. Blood surges into the false lumen, which can result in multiple organ damage due to hypoperfusion. The condition can quickly deteriorate into shock, hemodynamic instability and death. Emergent surgical repair remains the gold standard of care. Due to acuity of the illness preoperative evaluation is limited, immediate decisions have to be made by surgeons and postoperative adverse clinical outcome remains oftentimes of concern [1-8]. Without treatment mortality increases dramatically by the hour and has been reported as high as 1 to 3\% per hour during the first $24 \mathrm{~h}, 30 \%$ after one week, $80 \%$ after two weeks, and $90 \%$ at one year [9]. About $20 \%$ of patients with AAAD die before even reaching the hospital [9]. A recent multi-institutional study across all emergency rooms in Berlin, Germany from 2006 to 2016 
showed an incidence of AAAD of 5.24 cases in 100,000 visits per year but based on the city's autopsy results 50\% of AAAD had remained undetected [10]. Even with surgical repair mortality is high and ranges up to $16-27 \%$ within 30 -days [11-13]. We may be able to reduce mortality with advances in surgical strategies and perioperative critical care, but functional status and quality of life (QoL) in survivors are becoming an increasing concern since simply surviving surgery but then ending up in an overall devastating condition must not be a goal. Alterations in lifestyle and emotional state are common in survivors of AAAD and many patients are unable to return to their previous occupation [14]. Previous studies investigating the survival of AAAD patients have been published. But there is only scarce data on the effect of preoperative risk factors on clinical outcome of these patients. Hence, the aim of this study was to associate obvious preoperative conditions with a combined endpoint of undesirable adverse clinical outcome, that might guide clinicians in future decision-making.

\section{Materials and Methods}

\subsection{Study Design and Patient Population}

We performed a retrospective analysis of our Aortic Dissection Register, which included all consecutive 394 cases of AAAD who underwent emergent repair in moderate hypothermic cardiac arrest (MHCA) in our institution between 2001 and 2018. AAAD was defined as dissection of the aortic wall that involved the ascending aorta with extension to the arch or descending aorta, regardless of the site of the primary intimal tear. Variants with aortic intramural hematoma and intimal tears without hematoma as well as penetrating atherosclerotic ulcers were included. Diagnosis was generally established with emergent computed tomographic (CT) angiography of the chest, abdomen, and pelvis. Bedside transthoracic echocardiography was used to assess the presence of pericardial effusion and overall left ventricular function and in addition patients routinely underwent transesophageal echocardiography after induction of general anesthesia and endotracheal intubation in the operating room to evaluate heart valves for need for concomitant procedures. A combined endpoint of four clinical outcome parameters was defined as (1) 30-day versus hospital mortality, (2) new neurological deficit, (3) new acute renal insufficiency requiring postoperative renal replacement therapy, and (4) prolonged mechanical ventilation with need for tracheostomy. Follow-up was conducted in May 2020 and long-term survival was evaluated by information given by the registry office.

\subsection{Operative Technique and Postoperative Management}

All cases were performed by experienced senior surgeons under general anesthesia in supine position with standard hemodynamic monitoring. All patients underwent median sternotomy and longitudinal pericardiotomy with cardiopulmonary bypass $(\mathrm{CPB})$ in MHCA. The temperature probe was positioned in the nasopharynx and goal temperature was kept between 20 to $24^{\circ} \mathrm{C}$. From 2001 to 2010 arterial cannulation was achieved either by echocardiogram guided direct cannulation of the distal ascending aorta, the aortic arch, the apex, or either through the femoral or subclavian artery after surgical cut down. Starting in 2010 we gradually changed our standard approach for arterial cannulation to trans-atrial cannulation of the left ventricle via the right upper pulmonary vein [15]. The standard approach for venous drainage was cavoatrial cannulation with a common two-stage single venous cannula. Alternatively, we used echo guided cannulation of the femoral vein with a cannula extending into the right atrium or bicaval cannulation. Generally, we used retrograde injection of cold blood cardioplegic solution for myocardial protection after cross-clamping of the aorta. Bilateral antegrade cerebral perfusion with oxygenated cold blood $\left(18^{\circ} \mathrm{C}\right)$ was introduced through a balloon catheter inserted into the arch vessels with controlled flow pressure of 50-60 $\mathrm{mmHg}$.

The origin and extend of the intimal tear determined the need for supracoronary ascending aortic replacement, partial versus total arch replacement with reimplantation of head and neck arteries, frozen elephant trunk, need for associated coronary artery 
bypass grafting or Conduit/Bentall procedure with reimplantation of coronary arteries versus David operation. After suturing of the distal anastomosis, the perfusion cannula was directly inserted into the graft. The aortic air was removed by resuming retrograde perfusion via the venous cannula followed by slow antegrade perfusion and then CPB was restarted. Continuous $\mathrm{CO}_{2}$ insufflation was used in addition. After the establishment of the proximal anastomosis, transesophageal echocardiography was done to rule out remaining intracardiac air. After primary hemostasis was achieved, the chest was closed, and the patient was brought to the cardiac intensive care unit (ICU) for standard postoperative care.

Patients were assessed for neurological deficit routinely every hour while in the ICU and every eight hours after transfer to the floor. In case of a new deficit, CT head was performed followed by formal neurological evaluation and magnetic resonance imaging of the brain to confirm the diagnosis. Kidney function was assessed every hour while in the ICU and every eight hours on the floor. In case of acute renal insufficiency renal replacement therapy was initiated after evaluation by a nephrologist or in case of severe electrolyte disturbances emergently. Mechanical ventilation was weaned per standard postoperative protocol with a goal for liberation as soon as possible. Tracheostomy was performed if weaning from mechanical ventilation and extubation was not possible within 10-12 days postoperatively.

\subsection{Statistics}

Normally distributed continuous variables were presented as mean \pm standard deviation and compared by unpaired $t$-test. Categorical data were summarized as absolute (n) and relative (\%) frequencies and compared by $\mathrm{Chi}^{2}$-test or Fisher's exact test. Preand intraoperative variables were assessed for association with the combined endpoint by univariate analysis. 15-year survival was estimated by Kaplan-Meier curves. All tests were conducted 2-sided and a $p$-value of $\leq 0.05$ was considered statistically significant. Data were analyzed with IBM SPSS Statistics for Windows (Version 24.0).

\section{Results}

Total survival/ follow-up time averaged 3.2 years with follow-up completeness of $94 \%$. Follow-up was significantly shorter in the group who reached the combined endpoint, with 2.1 years versus 4.3 years, $p<0.001$.

\subsection{Preoperative Characteristics}

The combined endpoint was reached by $52.8 \%$. Patients who reached the endpoint had a significantly higher EuroSCORE II (7.5 versus 5.5, $p<0.001)$, higher incidence of coronary artery disease with previous percutaneous intervention $(9.2 \%$ versus $3.2 \%$, $p=0.016)$, higher incidence of neurological deficit upon presentation $(26.4 \%$ versus $11.8 \%$, $p<0.001)$, higher incidence of preoperative cardiopulmonary resuscitation $(14.4 \%$ versus $1.6 \%, p<0.001)$ and higher incidence of intubation before surgery $(16.9 \%$ versus $4.8 \%$, $p<0.001)$. There were no further significant differences with regard to clinical presentations between the groups. Table 1 shows detailed demographic and clinical characteristics of the study population.

\subsection{Intraoperative Characteristics}

Intraoperative characteristics are shown in Table 2. Patients who reached the combined endpoint also had significantly longer surgery duration (288 versus $256 \mathrm{~min}, p=0.001$ ), longer cardiopulmonary bypass times (180 versus $159 \mathrm{~min}, p<0.001)$, longer cross-clamp time (96 versus $84 \mathrm{~min}, p=0.010$ ), and longer circulatory arrest (39 versus $32 \mathrm{~min}, p<0.001$ ). The requirement for intraoperative transfusion of blood products was higher in the group who reached the combined endpoint (number of units of red blood cells 4 versus $2, p<0.001$, number of units of fresh frozen plasma 1.5 versus $0, p=0.031$, number of pools of platelets 2 (ranging from 5 to 0 ) versus 2 (ranging from 4 to 0 ), $p=0.002$ ). The need for total arch replacement was significantly higher in the group who reached the endpoint $(21.2 \%$ versus 
$8.1 \%, p<0.001)$. There were no differences between groups for all other surgical procedures such as single supracoronary replacement of the ascending aorta, partial arch replacement, Bentall operation, David Operation, Elephant trunk, associated coronary artery bypass grafting or cannulation site.

Table 1. Demographic and clinical characteristics of the study population.

\begin{tabular}{|c|c|c|c|c|}
\hline KERRYPNX & $\begin{array}{c}\text { All Patients } \\
(n=394)\end{array}$ & $\begin{array}{c}\text { Combined Endpoint } \\
(n=186 / 47.2 \%)\end{array}$ & $\begin{array}{c}\text { Combined Endpoint }=1 \\
(n=208 / 52.8 \%)\end{array}$ & $p$-Value \\
\hline \multirow{2}{*}{ Age, years } & $62.5 \pm 13.0$ & $61.7 \pm 14.0$ & $63.2 \pm 11.9$ & \multirow{2}{*}{0.567} \\
\hline & $63.0(53.0 ; 73.0)$ & $63.0(53.0 ; 71.3)$ & $63.5(53.3 ; 73.0)$ & \\
\hline Male gender & $256(65.0 \%)$ & $112(60.2 \%)$ & $144(69.2 \%)$ & 0.061 \\
\hline DeBakey classification, & & & & 0.108 \\
\hline DeBakey I & $292(78.5 \%)$ & $131(74.9 \%)$ & $161(81.7 \%)$ & \\
\hline DeBakey II & $80(21.5 \%)$ & $44(25.1 \%)$ & $36(18.3 \%)$ & \\
\hline Logistic EuroSCORE I & $28.7(18.1 ; 43.6)$ & $24.6(16.1 ; 39.7)$ & $31.8(18.7 ; 47.9)$ & 0.018 \\
\hline EuroSCORE II & $6.6(3.8 ; 13.3)$ & $5.5(3.6 ; 10.3)$ & $7.5(4.0 ; 15.9)$ & $<0.001$ \\
\hline Body mass index $\left[\mathrm{kg} / \mathrm{m}^{2}\right]$ & $26.2(23.9 ; 29.3)$ & $26.3(24.0 ; 29.4)$ & $26.1(23.8 ; 29.2)$ & 0.933 \\
\hline Body mass index $>30\left[\mathrm{~kg} / \mathrm{m}^{2}\right]$ & $79(20.1 \%)$ & $36(19.4 \%)$ & $43(20.8 \%)$ & 0.726 \\
\hline Arterial hypertension & $263(66.8 \%)$ & $125(67.2 \%)$ & $138(66.3 \%)$ & 0.857 \\
\hline Pulmonary hypertension & $7(1.8 \%)$ & $4(2.2 \%)$ & $3(1.4 \%)$ & 0.712 \\
\hline Type 2 Diabetes mellitus & $20(5.1 \%)$ & $7(3.8 \%)$ & $13(6.3 \%)$ & 0.262 \\
\hline Insulin dependent & $6(1.5 \%)$ & $3(1.6 \%)$ & $3(1.4 \%)$ & 1.000 \\
\hline Hyperlipoproteinaemia & $42(10.7 \%)$ & $22(11.8 \%)$ & $20(9.6 \%)$ & 0.477 \\
\hline Creatinine at admission $>200[\mu \mathrm{mol} / \mathrm{L}]$ & $17(4.6 \%)$ & $6(3.4 \%)$ & $11(5.8 \%)$ & 0.270 \\
\hline Chronic renal insufficiency & $46(11.7 \%)$ & $16(8.6 \%)$ & $30(14.4 \%)$ & 0.072 \\
\hline Decompensated renal insufficiency & $9(2.3 \%)$ & $3(1.6 \%)$ & $6(2.9 \%)$ & 0.510 \\
\hline $\begin{array}{l}\text { Renal replacement therapy } \\
\text { ("chron Dialyse") }\end{array}$ & $7(1.8 \%)$ & $4(2.2 \%)$ & $3(1.4 \%)$ & 0.712 \\
\hline COPD & $26(6.6 \%)$ & $13(7.0 \%)$ & $13(6.3 \%)$ & 0.768 \\
\hline Peripheral vascular disease & $15(3.8 \%)$ & $7(3.8 \%)$ & $8(3.8 \%)$ & 0.966 \\
\hline Smoking & $75(19.1 \%)$ & $37(19.9 \%)$ & $38(18.4 \%)$ & 0.699 \\
\hline Coronary heart disease & $68(17.3 \%)$ & $25(13.4 \%)$ & $43(20.7 \%)$ & 0.058 \\
\hline \multicolumn{5}{|l|}{ Heart rhythm } \\
\hline Sinus rhythm & $328(83.2 \%)$ & $159(85.5 \%)$ & $169(81.3 \%)$ & 0.261 \\
\hline Atrial fibrillation & $54(13.7 \%)$ & $22(11.8 \%)$ & $32(15.4 \%)$ & 0.305 \\
\hline LVEF $(\%)$ & $60(55 ; 70)$ & $60(56 ; 70)$ & $60(55 ; 70)$ & 0.200 \\
\hline Previous PCI & $25(6.4 \%)$ & $6(3.2 \%)$ & $19(9.2 \%)$ & 0.016 \\
\hline Previous cardiac surgery & $36(9.1 \%)$ & $21(11.3 \%)$ & $15(7.2 \%)$ & 0.161 \\
\hline Previous CABG & $12(3.0 \%)$ & $5(2.7 \%)$ & $7(3.4 \%)$ & 0.696 \\
\hline IABP/ECLS & $5(1.3 \%)$ & $0(0.0 \%)$ & $5(2.4 \%)$ & 0.063 \\
\hline Pericardial tamponade & $71(18.1 \%)$ & $29(15.6 \%)$ & $42(20.3 \%)$ & 0.227 \\
\hline Marfan syndrome & $11(2.8 \%)$ & $7(3.8 \%)$ & $4(1.9 \%)$ & 0.272 \\
\hline Bicuspid aortic valve & $18(4.7 \%)$ & $8(4.4 \%)$ & $10(4.9 \%)$ & 0.849 \\
\hline Aortic valve vitium & & & & 0.987 \\
\hline Aortic valve stenosis & $10(2.6 \%)$ & $5(2.7 \%)$ & $5(2.5 \%)$ & 1.000 \\
\hline Aortic valve insufficiency & $133(35.1 \%)$ & $65(35.7 \%)$ & $68(34.5 \%)$ & 0.807 \\
\hline $\begin{array}{l}\text { Combined Aortic valve vitium at Aortic } \\
\text { valve replacement }\end{array}$ & $6(1.6 \%)$ & $3(1.6 \%)$ & $3(1.5 \%)$ & 1.000 \\
\hline Neurological deficits & $77(19.5 \%)$ & $22(11.8 \%)$ & $55(26.4 \%)$ & $<0.001$ \\
\hline
\end{tabular}


Table 1. Cont.

\begin{tabular}{|c|c|c|c|c|}
\hline KERRYPNX & $\begin{array}{l}\text { All Patients } \\
(n=394)\end{array}$ & $\begin{array}{c}\text { Combined Endpoint } \\
(n=186 / 47.2 \%)\end{array}$ & $\begin{array}{c}\text { Combined Endpoint } \\
(n=208 / 52.8 \%)\end{array}$ & $p$-Value \\
\hline \multicolumn{5}{|l|}{ Clinical presentation } \\
\hline Acute myocardial infarction $(\leq 48 \mathrm{~h})$ & $14(3.6 \%)$ & $4(2.2 \%)$ & $10(4.8 \%)$ & 0.155 \\
\hline Cardiogenic shock & $30(7.6 \%)$ & $10(5.4 \%)$ & $20(9.7 \%)$ & 0.110 \\
\hline $\mathrm{CPR}(\leq 48 \mathrm{~h})$ & $33(8.4 \%)$ & $3(1.6 \%)$ & $30(14.4 \%)$ & $<0.001$ \\
\hline Transfer from intensive care unit & $47(11.9 \%)$ & $16(8.6 \%)$ & $31(14.9 \%)$ & 0.054 \\
\hline Intubated at admission & $44(11.2 \%)$ & $9(4.8 \%)$ & $35(16.9 \%)$ & $<0.001$ \\
\hline
\end{tabular}

Table 2. Operative data.

\begin{tabular}{|c|c|c|c|c|}
\hline & $\begin{array}{c}\text { All Patients } \\
(n=394)\end{array}$ & $\begin{array}{c}\text { Combined Endpoint } \\
(n=186 / 47.2 \%)\end{array}$ & $\begin{array}{c}\text { Combined Endpoint }=1 \\
(n=208 / 52.8 \%)\end{array}$ & $p$-Value \\
\hline Length of surgery [min] & $275(227 ; 340)$ & $256(218 ; 311)$ & $288(233 ; 358)$ & 0.001 \\
\hline Cardiopulmonary bypass time [min] & $167(136 ; 212)$ & $159(130 ; 199)$ & $180(140 ; 228)$ & $<0.001$ \\
\hline Cross-clamp time $[\mathrm{min}]$ & $92(71 ; 132)$ & $84(65 ; 130)$ & $96(75 ; 134)$ & 0.010 \\
\hline Circulatory arrest [min] & $35(26 ; 50)$ & $32(24 ; 42)$ & $39(28 ; 60)$ & $<0.001$ \\
\hline Number of packed red blood cells, unit & $2.5(0-16)$ & $2(0-16)$ & $4(0-16)$ & $<0.001$ \\
\hline Number of fresh frozen plasma, unit & $0(0-21)$ & $0(0-16)$ & $1.5(0-21)$ & 0.031 \\
\hline Number of platelets, unit & $2(0-5)$ & $2(0-4)$ & $2(0-5)$ & 0.002 \\
\hline \multicolumn{5}{|l|}{ Surgical procedure } \\
\hline $\begin{array}{l}\text { Single supracoronary replacement of the } \\
\text { ascending aorta }\end{array}$ & $187(47.5 \%)$ & $87(46.8 \%)$ & $100(48.1 \%)$ & 0.796 \\
\hline Partial arch replacement & $94(23.9 \%)$ & $50(27.0 \%)$ & $44(21.2 \%)$ & 0.173 \\
\hline Total arch replacement & $59(15.0 \%)$ & $15(8.1 \%)$ & $44(21.2 \%)$ & $<0.001$ \\
\hline Conduit/Bentall operation & $72(18.3 \%)$ & $35(18.8 \%)$ & $37(17.8 \%)$ & 0.792 \\
\hline David operation & $24(6.1 \%)$ & $15(8.1 \%)$ & $9(4.3 \%)$ & 0.121 \\
\hline Elephant-trunk & $9(2.3 \%)$ & $2(1.1 \%)$ & $7(3.4 \%)$ & 0.181 \\
\hline Associated with Aortic valve replacement & $65(16.5 \%)$ & $30(16.1 \%)$ & $35(16.8 \%)$ & 0.852 \\
\hline Associated with CABG & $29(7.4 \%)$ & $9(4.8 \%)$ & $20(9.6 \%)$ & 0.070 \\
\hline TEVAR(EVAR) & $27(6.9 \%)$ & $10(5.4 \%)$ & $17(8.2 \%)$ & 0.267 \\
\hline Arterial cannulation & & & & 0.612 \\
\hline Femoral artery & $62(15.7 \%)$ & $30(16.1 \%)$ & $32(15.4 \%)$ & 0.839 \\
\hline Ascending aorta & $83(21.1 \%)$ & $33(17.7 \%)$ & $50(24.0 \%)$ & 0.126 \\
\hline Aortic arch & $9(2.3 \%)$ & $4(2.2 \%)$ & $5(2.4 \%)$ & 1.000 \\
\hline Subclavian artery & $1(0.3 \%)$ & $0(0.0 \%)$ & $1(0.5 \%)$ & 1.000 \\
\hline Apex & $5(1.3 \%)$ & $2(1.1 \%)$ & $3(1.4 \%)$ & 1.000 \\
\hline Pulmonary vein & $234(59.4 \%)$ & $117(62.9 \%)$ & $117(56.3 \%)$ & 0.179 \\
\hline \multicolumn{5}{|l|}{ Venous cannulation } \\
\hline Right atrium & $382(97.2 \%)$ & $183(98.4 \%)$ & $199(96.1 \%)$ & 0.328 \\
\hline Bicaval & $3(0.8 \%)$ & $0(0.0 \%)$ & $3(1.4 \%)$ & 0.177 \\
\hline Femoral vein & $8(2.0 \%)$ & $3(1.6 \%)$ & $5(2.4 \%)$ & 0.727 \\
\hline
\end{tabular}

\subsection{Postoperative Data and Outcome}

Postoperative data and outcomes are shown in Table 3. Mortality was higher and complications were more common in the group who reached the combined end point. 7 -day mortality was $21.6 \%$ versus $0 \%, p<0.001$. Hospital mortality was $30.8 \%$ versus $0 \%$, $p<0.001$. Causes of death were cardiac $53 \%$, multiple organ failure in $43 \%$, cerebral $9 \%$, and sepsis $3 \%$. The group who reached the endpoint had a significantly longer stay in 
the intensive care unit (10 days versus 4 days, $p<0.001$ ), larger amount of postoperative drainage loss $(1030 \mathrm{~mL}$ versus $750 \mathrm{~mL}, p<0.001$, greater need for postoperative blood transfusions $(83.7 \%$ versus $64.5 \%$ of patients, $p<0.001)$, fresh frozen plasma transfusions $(60.1 \%$ versus $40.3 \%, p<0.001)$ and platelet transfusions $(55.9 \%$ versus $37.6 \%, p<0.001)$, as well as higher incidence of re-thoracotomy $(26.9 \%$ versus $8.1 \%, p<0.001)$. They also had a greater need for postoperative balloon pump and/or extracorporeal life support $(5.1 \%$ versus $0.5 \%, p=0.008)$, reintubation $(27.9 \%$ versus $5.9 \%, p<0.001)$, prolonged mechanical ventilation (189 h versus $24 \mathrm{~h}, p<0.001)$ with need for tracheostomy $(47.6 \%$ versus $0 \%, p<0.001)$, readmission to the intensive care unit $(13.5 \%$ versus $4.3 \%, p=0.002)$, bacteremia/sepsis $(8.7 \%$ versus $0.5 \%, p<0.001)$, bronchopulmonary infection $(22.1 \%$ versus $6.5 \%, p<0.001)$, cardiac arrest $(11.1 \%$ versus $2.2 \%, p<0.001)$, new neurological deficit consistent with TIA/stroke ( $45.2 \%$ versus $0 \%, p<0.001)$, myocardial infarction $(2.9 \%$ versus $0 \%, p=0.032)$, and acute renal insufficiency with need for renal replacement therapy $(41.3 \%$ versus $0 \%, p<0.001)$. While several parameters were less common in the group that reached the endpoint, they showed no statistical significance. Those were postoperative delirium $(15.9 \%$ versus $21.1 \%)$, sternal wound infections $(1.0 \%$ versus $2.2 \%)$ and atrial fibrillation $(10.7 \%$ versus $10.2 \%)$.

Table 3. Postoperative data and outcomes.

\begin{tabular}{|c|c|c|c|c|}
\hline & $\begin{array}{l}\text { All Patients } \\
\quad(n=394)\end{array}$ & $\begin{array}{c}\text { Combined Endpoint }=0 \\
(n=186 / 47.2 \%)\end{array}$ & $\begin{array}{l}\text { Combined Endpoint }=1 \\
\quad(n=208 / 52.8 \%)\end{array}$ & $p$-Value \\
\hline 48 h-drainage loss [mL] & $900(500 ; 1513)$ & $750(350 ; 1200)$ & $1030(650 ; 1878)$ & $<0.001$ \\
\hline Postoperative blood transfusion & $290(74.6 \%)$ & $120(64.5 \%)$ & $170(83.7 \%)$ & $<0.001$ \\
\hline Postoperative fresh frozen plasma & $197(50.6 \%)$ & $75(40.3 \%)$ & $122(60.1 \%)$ & $<0.001$ \\
\hline Postoperative platelets & $183(47.2 \%)$ & $70(37.6 \%)$ & $113(55.9 \%)$ & $<0.001$ \\
\hline 24 h-Number of packed red blood cells, unit, & $1(0-17)$ & $0(0-17)$ & $1(0-15)$ & 0.029 \\
\hline 24 h-Number of fresh frozen plasma, unit, & $0(0-24)$ & $0(0-24)$ & $0.5(0-23)$ & $<0.001$ \\
\hline 24 h-Number of platelets, unit, & $0(0-10)$ & $0(0-5)$ & $0(0-10)$ & $<0.001$ \\
\hline Total number of packed red blood cells, unit & $4(0-56)$ & $2(0-38)$ & $6(0-56)$ & $<0.001$ \\
\hline Total number of fresh frozen plasma, unit & $1(0-76)$ & $0(0-36)$ & $4(0-76)$ & $<0.001$ \\
\hline Total number of platelets, unit & $0(0-20)$ & $0(0-9)$ & $1(0-20)$ & $<0.001$ \\
\hline IABP/ECLS & $11(2.9 \%)$ & $1(0.5 \%)$ & $10(5.1 \%)$ & 0.008 \\
\hline Reintubation & $69(17.5 \%)$ & $11(5.9 \%)$ & $58(27.9 \%)$ & $<0.001$ \\
\hline Tracheotomy & $99(25.1 \%)$ & $0(0.0 \%)$ & $99(47.6 \%)$ & $<0.001$ \\
\hline Re-admission to the ICU & $36(9.2 \%)$ & $8(4.3 \%)$ & $28(13.5 \%)$ & 0.002 \\
\hline Postoperative delirium & $72(18.4 \%)$ & $39(21.1 \%)$ & $33(15.9 \%)$ & 0.190 \\
\hline Postoperative myocardial infarction & $6(1.5 \%)$ & $0(0.0 \%)$ & $6(2.9 \%)$ & 0.032 \\
\hline TIA/Stroke & $94(23.9 \%)$ & $0(0.0 \%)$ & $94(45.2 \%)$ & $<0.001$ \\
\hline $\mathrm{CPR}$ & $27(6.9 \%)$ & $4(2.2 \%)$ & $23(11.1 \%)$ & $<0.001$ \\
\hline Bronchopulmonary infection & $58(14.7 \%)$ & $12(6.5 \%)$ & $46(22.1 \%)$ & $<0.001$ \\
\hline Bacteriaemia/sepsis & $19(4.8 \%)$ & $1(0.5 \%)$ & $18(8.7 \%)$ & $<0.001$ \\
\hline Rethoracotomy & $71(18.0 \%)$ & $15(8.1 \%)$ & $56(26.9 \%)$ & $<0.001$ \\
\hline Sternal wound infection/VAC revision & $6(1.5 \%)$ & $4(2.2 \%)$ & $2(1.0 \%)$ & 0.431 \\
\hline New -onset of Hemodialysis & $85(21.7 \%)$ & $0(0.0 \%)$ & $85(41.3 \%)$ & $<0.001$ \\
\hline Atrial fibrillation & $41(10.5 \%)$ & $19(10.2 \%)$ & $22(10.7 \%)$ & 0.881 \\
\hline Ventilation time $[\mathrm{h}]$ & $69(20 ; 209)$ & $24(15 ; 57)$ & $189(81 ; 387)$ & $<0.001$ \\
\hline ICU time $[\mathrm{d}]$ & $6(2 ; 12)$ & $4(2 ; 6)$ & $10(4 ; 18)$ & $<0.001$ \\
\hline Postoperative days & $10(7 ; 19)$ & $9(7 ; 13)$ & $13(7 ; 23)$ & $<0.001$ \\
\hline 7 d-Mortality & $45(11.4 \%)$ & $0(0.0 \%)$ & $45(21.6 \%)$ & $<0.001$ \\
\hline Hospital Mortality & $64(16.2 \%)$ & $0(0.0 \%)$ & $64(30.8 \%)$ & $<0.001$ \\
\hline Cardiac death & $34(53.1 \%)$ & $0(0.0 \%)$ & $34(53.1 \%)$ & - \\
\hline Cerebral death & $6(9.4 \%)$ & $0(0.0 \%)$ & $6(9.4 \%)$ & - \\
\hline Sepsis & $2(3.1 \%)$ & $0(0.0 \%)$ & $2(3.1 \%)$ & - \\
\hline MOF & $22(34.4 \%)$ & $0(0.0 \%)$ & $22(34.4 \%)$ & - \\
\hline
\end{tabular}




\subsection{Risk Factors for Combined Endpoint}

Independent preoperative risk factors to reach the combined endpoint of mortality, new neurological deficit, prolonged mechanical ventilation with need for tracheostomy and acute renal insufficiency with need for renal replacement therapy were assessed with multivariable logistic regression analysis as shown in Table 4. Significant were coronary heart disease ( $p=0.021$, OR 2.122, CI 1.1-4.0), presence of a neurological deficit $(p<0.001$, OR 3.6, CI 1.98-6.5), preoperative need for cardiopulmonary resuscitation $(p=0.001$, OR 8.99, CI 2.5-32.3) and need for intubation on admission ( $p=0.033$, OR 2.5, CI 1.1-5.9).

Table 4. Multivariable analysis of risk factors for the combined endpoint.

\begin{tabular}{cccc}
\hline Variable & $p$ & Odds Ratio & Confidence Interval \\
\hline Coronary heart disease & 0.021 & 2.122 & $1.118-4.028$ \\
Neurological deficits & $<0.001$ & 3.598 & $1.985-6.521$ \\
CPR & 0.001 & 8.993 & $2.501-32.343$ \\
Intubated at admission & 0.033 & 2.512 & $1.077-5.861$ \\
\hline
\end{tabular}

\subsection{Survival Curve}

Figure 1 shows the Kaplan-Meier survival curve of patients who did and did not reach the combined endpoint with a follow-up time of 15 years. The group who reached the endpoint had significantly decreased 15-year survival, however, it is notable that curves are almost parallel, after the first 30-days, indicating that the highest rate of death occurs in the immediate postoperative period.

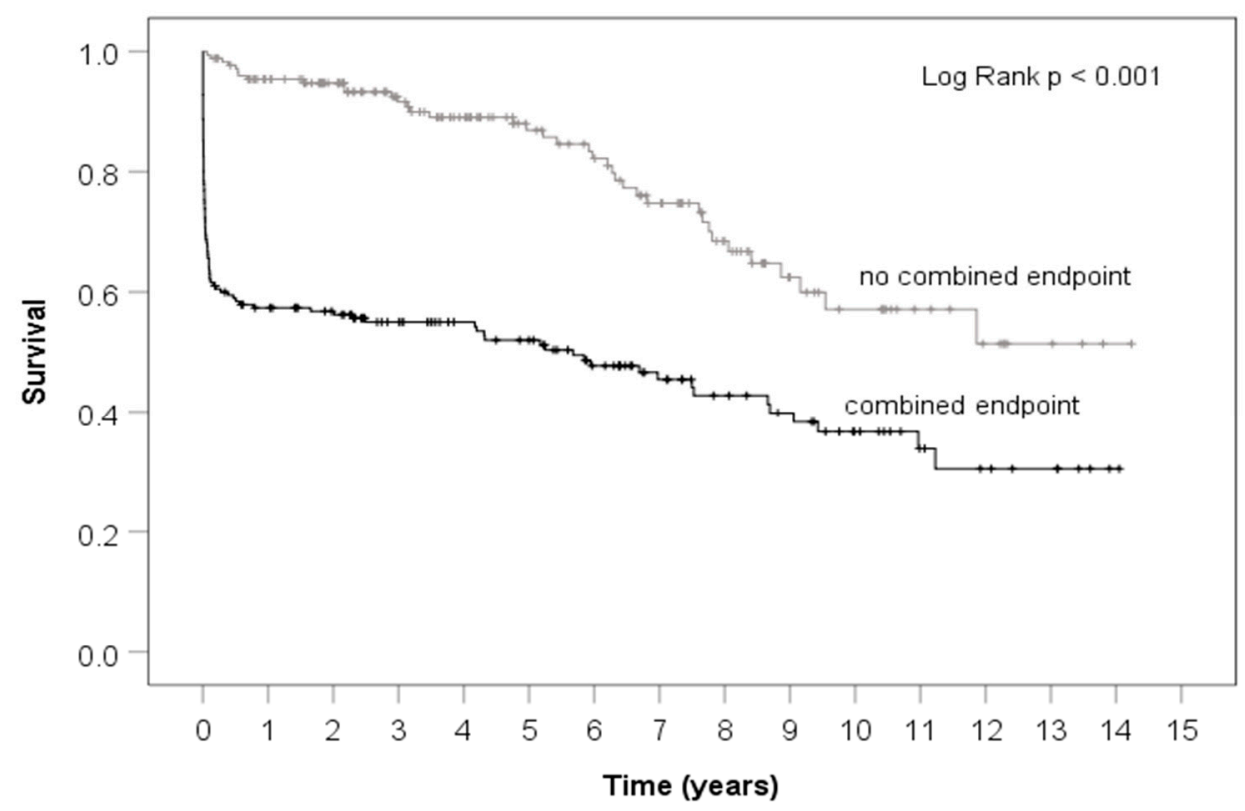

Figure 1. Survival curves of patients with and without reaching the combined endpoint.

\section{Discussion}

It seems remarkable that the majority of patients who had complications did not just have one but multiple. Taken all facts into account, $52.8 \%$ of the patients in our population had an undesirable outcome.

Many previous studies have already evaluated risk factors for postoperative survival $[9,16-19]$, but the universal ethical question remains in which high risk cases withholding surgery would provide less harm than performing it, since over $50 \%$ of survivors may have to tolerate devastating conditions on long term ventilation with tracheostomy, long-term dialysis and a severe neurological deficit. IRAD data indicated a mortality of $58 \%$ among those not receiving surgery, typically because of advanced age and comorbidity [20]. 
The intention of this study was to assess if undesirable post-operative outcome was associated with certain parameters present on presentation, to help eventually develop a strategy to know for which patient surgery is likely harmful. Accordingly, we chose a combination of severe debilitating complications as endpoint.

A common assumption is that patients with multiple underlying medical conditions such as hypertension, diabetes, hyperlipidemia, chronic kidney disease, smoking or previous cardiac surgery have adverse outcome [2]. However, in our study, the group who reached the combined endpoint had no higher incidence of such diagnoses, despite a higher EuroSCORE II. Therefore, the proposal is, that risk factors for developing aortic dissection are not applicable for suffering poor post-operative outcome. According to data from the Swedish National Diabetes Register patients with type 2 diabetes actually had significantly less risk of aortic aneurysm, dissection and reduced mortality after hospitalization compared to matched healthy controls. The authors hypothesized that glycated cross-links in aortic tissue may play a protective role in the progression of aortic diseases [21]. The previously published analysis of our database suggested that mortality was multifactorial and especially age, previous cardiac surgery, preoperative cardiopulmonary resuscitation, blood transfusion, and postoperative renal failure were considered risk factors [6].

According to our current data, the clinical condition in which the patient arrives preoperatively is predictive of poor outcome. Other authors showed as well that inhospital adverse outcome was associated with the presence of lower limb hypoperfusion symptoms prior to surgery [2]. Since time is such an essential part, prompt diagnosis and referral to immediate surgical repair remain the main goal. Michael DeBakey once stated: "no physician can diagnose a condition he never thinks about". An analysis from the International Registry of Acute Aortic Dissection (IRAD) indicates that the median time from emergency department presentation to definitive diagnosis of acute aortic dissection is $4.3 \mathrm{~h}$, with an additional $4 \mathrm{~h}$ between diagnosis and surgical intervention for type A patients [22].

The response time for emergency medical services is legally regulated in Germany and should not exceed 12 min from alarm to arrival in our federal state [23], but even in densely populated areas averages 8-10 min. Emergency physicians ride on the ambulance and can make an immediate assessment. If AAAD is suspected, the physician alarms the emergency room personnel to have imaging available immediately on arrival, as well as the cardiovascular surgeon on stand-by. Despite these seemingly ideal conditions, analysis of the German Registry for Acute Aortic Dissection Type A including 2137 patients by Boening et al., revealed an overall 30-day mortality of $16.9 \%$ and new neurologic dysfunction postoperatively in $9.5 \%$ [11]. While our mortality coincides well with the national level, our rate of neurological complications seems to be higher ranging up to $23.9 \%$. In another single center retrospective analysis by Haldenwang et al., the 30-day mortality rate was $16.4 \%$. In their population $33.6 \%$ suffered transient neurological dysfunction and $16.4 \%$ had a postoperative stroke [5]. They also looked at a combined adverse outcome defined as stroke and 30-day mortality and found high body mass index, preoperative hypoperfusion syndrome, and left ventricular ejection fraction $<50 \%$ to be independent predictors. Our results indicate a higher incidence of cardiopulmonary resuscitation within $48 \mathrm{~h}$ before surgery and preoperative mechanical ventilation in the combined endpoint group, but there was no higher incidence in the presence of IABP/ ECLS, cardiogenic shock, pericardial tamponade or decompensated renal insufficiency. There was also no higher prevalence of Marfan Syndrome or difference in average left ventricular ejection fraction.

Current risk assessment scores don't seem to provide an accurate answer in AAAD, especially EuroSCORE II appears to underestimate mortality. Our current analysis does not evaluate postoperative QoL in such circumstances. A previous investigation within our group found however, that the QoL scores were lower one year after emergent surgery for AAAD compared to the general, age-matched population in Germany especially regarding pain score and social functioning [3]. 
With growing socioeconomic and financial pressure in hospitals and healthcare systems, early identification of patients at risk for prolonged length of hospital stay with needs for advanced therapies is also essential. It was no surprise that patients who reached the combined endpoint had significantly longer stays on the ventilator, in the intensive care unit as well as in the hospital compared to those who did not reach the endpoint.

Our results stress again the importance of early diagnosis of AAAD and immediate referral to a facility capable to operate immediately, since the clinical condition on arrival plays such an important role as prognostic marker.

This study is designed as single-center retrospective review of an internal database and not a randomized prospective trial. Information was obtained from our institutional database. Data were entered by staff physicians during the patients' hospitalizations. Therefore, data may be subject to bias. From our data it remains unclear if and how our change of strategy regarding atrial canulation may have influenced the outcome.

\section{Conclusions}

We showed, in 15-year follow up, that relevant risk factors for adverse postoperative clinical outcome are rather related to the clinical condition in which the patient arrives preoperatively, than preexisting medical illnesses widely assumed to be responsible for poor outcome. This supports prioritizing immediate surgical attention to patients, before they may otherwise progress to hemodynamic instability and hypoperfusion even if they have underlying medical conditions or advanced age. The ethical dilemma arises when patients arrive at the hospital with already existing hypoperfusion, ongoing cardiopulmonary resuscitation or even intubation. In those cases, our data suggest that physicians may recommend either non-surgical treatment due to extremely poor chances for acceptable outcome or have a detailed discussion with patient and families of what to expect.

Author Contributions: Conceptualization, A.H. and J.C.; methodology, C.F.; software, T.P.; validation, M.G., G.E. and M.S.; formal analysis, C.F.; investigation, P.K., A.H.; resources, M.F.; data curation, C.F.; writing —original draft preparation, M.F.; writing—review and editing, A.H.; visualization, C.F.; supervision, J.C.; All authors have read and agreed to the published version of the manuscript.

Funding: This research received no external funding.

Institutional Review Board Statement: This study was approved by the Local Ethics Committee (Christian-Albrechts-University Kiel, Schwanenweg 20, D-24105 Kiel, Referral number: D417/17XXX).

Informed Consent Statement: Patient consent to participate in this study was waived due to the retrospective design. However, written informed consent for surgery was obtained from all surviving patients included into this study.

Conflicts of Interest: The authors declare no conflict of interest.

\section{References}

1. Di Eusanio, M.; Trimarchi, S.; Patel, H.J.; Hutchison, S.; Suzuki, T.; Peterson, M.D.; di Bartolomeo, R.; Folesani, G.; Pyeritz, R.E.; Braverman, A.C.; et al. Clinical presentation, management, and short-term outcome of patients with type A acute dissection complicated by mesenteric malperfusion: Observations from the International Registry of Acute Aortic Dissection. J. Thorac. Cardiovasc. Surg. 2013, 145, 385-390.e1. [CrossRef]

2. Wei, J.; Chen, Z.; Zhang, H.; Sun, X.; Qian, X.; Yu, C. In-hospital major adverse outcomes of acute Type A aortic dissection. Eur. J. Cardio Thorac. Surg. 2019, 55, 345-350. [CrossRef] [PubMed]

3. Jussli-Melchers, J.; Panholzer, B.; Friedrich, C.; Broch, O.; Renner, J.; Schöttler, J.; Rahimi, A.; Cremer, J.; Schoeneich, F.; Haneya, A. Long-term outcome and quality of life following emergency surgery for acute aortic dissection type A: A comparison between young and elderly adults. Eur. J. Cardio Thorac. Surg. 2016, 51, 465-471. [CrossRef]

4. Friedrich, C.; Salem, M.A.; Puehler, T.; Hoffmann, G.; Lutter, G.; Cremer, J.; Haneya, A. Sex-specific risk factors for early mortality and survival after surgery of acute aortic dissection type a: A retrospective observational study. J. Cardiothorac. Surg. 2020, 15, 1-12. [CrossRef]

5. Haldenwang, P.L.; Wahlers, T.; Himmels, A.; Wippermann, J.; Zeriouh, M.; Kröner, A.; Kuhr, K.; Strauch, J.T. Evaluation of risk factors for transient neurological dysfunction and adverse outcome after repair of acute type A aortic dissection in 122 consecutive patients. Eur. J. Cardio Thorac. Surg. 2012, 42, e115-e120. [CrossRef] 
6. Salem, M.; Friedrich, C.; Thiem, A.; Huenges, K.; Puehler, T.; Cremer, J.; Haneya, A. Risk Factors for Mortality in Acute Aortic Dissection Type A: A Centre Experience Over 15 Years. Thorac. Cardiovasc. Surg. 2021, 69, 322-328. [CrossRef]

7. Conzelmann, L.O.; Weigang, E.; Mehlhorn, U.; Abugameh, A.; Hoffmann, I.; Blettner, M.; Etz, C.D.; Czerny, M.; Vahl, C.F. Mortality in patients with acute aortic dissection type A: Analysis of pre- and intraoperative risk factors from the German Registry for Acute Aortic Dissection Type A (GERAADA). Eur. J. Cardio Thorac. Surg. 2016, 49, e44-e52. [CrossRef] [PubMed]

8. Caus, T.; Frapier, J.M.; Giorgi, R.; Aymard, T.; Riberi, A.; Albat, B.; Chaptal, P.A.; Mesana, T. Clinical outcome after repair of acute type A dissection in patients over 70 years-old. Eur. J. Cardio Thorac. Surg. 2002, 22, 211-217. [CrossRef]

9. Tsai, T.T.; Evangelista, A.; Nienaber, C.A.; Trimarchi, S.; Sechtem, U.; Fattori, R.; Myrmel, T.; Pape, L.; Cooper, J.V.; Smith, D.E.; et al. Long-Term Survival in Patients Presenting with Type A Acute Aortic Dissection: Insights from the International Registry of Acute Aortic Dissection (IRAD). J. Circ. 2006, 114, I-350-I-356. [CrossRef] [PubMed]

10. Wundram, M.; Falk, V.; Eulert-Grehn, J.-J.; Herbst, H.; Thurau, J.; Leidel, B.A.; Göncz, E.; Bauer, W.; Habazettl, H.; Kurz, S.D. Incidence of acute type A aortic dissection in emergency departments. Sci. Rep. 2020, 10, 1-6. [CrossRef]

11. Karck, M.; Conzelmann, L.; Easo, J.; Krüger, T.; Rylski, B.; Weigang, E.; Boening, A. German Registry for Acute Aortic Dissection Type A: Structure, Results, and Future Perspectives. Thorac. Cardiovasc. Surg. 2016, 65, 077-084. [CrossRef] [PubMed]

12. Easo, J.; Weigang, E.; Hölzl, P.P.; Horst, M.; Hoffmann, I.; Blettner, M.; Dapunt, O.E. Influence of operative strategy for the aortic arch in DeBakey type I aortic dissection: Analysis of the German Registry for Acute Aortic Dissection Type A. J. Thorac. Cardiovasc. Surg. 2012, 144, 617-623. [CrossRef] [PubMed]

13. Rylski, B.; Hoffmann, I.; Beyersdorf, F.; Suedkamp, M.; Siepe, M.; Nitsch, B.; Blettner, M.; Borger, M.; Weigang, E. Acute Aortic Dissection Type A: Age-related management and outcomes reported in the german registry for acute aortic dissection type a (GERAADA) of over 2000 patients. Ann. Surg. 2014, 259, 598-604. [CrossRef]

14. Chaddha, A.; Kline-Rogers, E.; Braverman, A.C.; Erickson, S.R.; Jackson, E.A.; Franklin, B.A.; Bs, E.M.W.; Jabara, J.T.; Ms, D.G.M.; Eagle, K.A. Survivors of Aortic Dissection: Activity, Mental Health, and Sexual Function. Clin. Cardiol. 2015, 38, 652-659. [CrossRef]

15. Rahimi-Barfeh, A.; Grothusen, C.; Haneya, A.; Schöttler, J.; Eide, A.M.; Erdmann, M.; Friedrich, C.; Hoffmann, G.; Cremer, J.; Schoeneich, F. Transatrial Cannulation of the Left Ventricle for Acute Type A Aortic Dissection: A 5-Year Experience. Ann. Thorac. Surg. 2016, 101, 1753-1758. [CrossRef] [PubMed]

16. Mehta, R.H.; Suzuki, T.; Hagan, P.G.; Bossone, E.; Gilon, D.; Llovet, A.; Maroto, L.C.; Cooper, J.V.; Smith, D.E.; Armstrong, W.F.; et al. Predicting Death in Patients with Acute Type A Aortic Dissection. J. Circ. 2002, 105, $200-206$. [CrossRef]

17. Bossone, E.; Rampoldi, V.; Nienaber, C.; Trimarchi, S.; Ballotta, A.; Cooper, J.V.; Smith, D.; Eagle, K.; Mehta, R.H. Usefulness of pulse deficit to predict in-hospital complications and mortality in patients with acute type A aortic dissection. Am. J. Cardiol. 2002, 89, 851-855. [CrossRef]

18. Rampoldi, V.; Trimarchi, S.; Eagle, K.A.; Nienaber, C.A.; Oh, J.K.; Bossone, E.; Myrmel, T.; Sangiorgi, G.; De Vincentiis, C.; Cooper, J.V.; et al. Simple Risk Models to Predict Surgical Mortality in Acute Type A Aortic Dissection: The International Registry of Acute Aortic Dissection Score. Ann. Thorac. Surg. 2007, 83, 55-61. [CrossRef]

19. Yang, G.; Zhou, Y.; He, H.; Pan, X.; Li, X.; Chai, X. A nomogram for predicting in-hospital mortality in acute type A aortic dissection patients. J. Thorac. Dis. 2020, 12, 264-275. [CrossRef] [PubMed]

20. Hagan, P.G.; Nienaber, C.A.; Isselbacher, E.M.; Bruckman, D.; Karavite, D.J.; Russman, P.L.; Evangelista, A.; Fattori, R.; Suzuki, T.; Oh, J.K.; et al. The International Registry of Acute Aortic Dissection (IRAD). JAMA 2000, 283, 897-903. [CrossRef]

21. Avdic, T.; Franzén, S.; Zarrouk, M.; Acosta, S.; Nilsson, P.; Gottsäter, A.; Svensson, A.; Gudbjörnsdottir, S.; Eliasson, B. Reduced Long-Term Risk of Aortic Aneurysm and Aortic Dissection Among Individuals with Type 2 Diabetes Mellitus: A Nationwide Observational Study. J. Am. Hear. Assoc. 2018, 7, e007618. [CrossRef] [PubMed]

22. Lloyd-Jones, D.M. Cardiovascular health and protection against CVD: More than the sum of the parts? J. Circ. 2014, 130, 1671-1673. [CrossRef] [PubMed]

23. Gesetze-Rechtsprechung Schleswig-Holstein RettDGDV SH 2019 | Landesnorm Schleswig-Holstein I Gesamtausgabe | Landesverordnung zur Durchführung des Schleswig-Holsteinischen Rettungsdienstgesetzes (SHRDG-DVO) vom 4. Dezember 2018 | Gültig von: 01.01.2019 Gültig bis: 31.12.2023. Available online: http:/ / www.gesetze-rechtsprechung.sh.juris.de/jportal/?quelle=jlink\&query=RettDGDV+ SH\&psml=bsshoprod.psml\&max=true\&aiz=true (accessed on 8 September 2020). 